\section{- Clinical Pharmacist Intervention in a Primary Care Medical Group Reduces Financial Losses}

Global capitation contracts based on a percent of premium and including pharmacy benefit costs have pushed physician-hospital organizations (PHOs) into bankruptcy, including large PHOs with as many as 150,000 members enrolled under such risk contracts. ${ }^{1}$ A recent report from an academic $\mathrm{PHO}$ with more than 1,000 physicians operating under a global capitation contract showed that providing primary care physicians (PCPs) with information resulted in a $22 \%$ reduction in the use of highcost drugs. ${ }^{2}$ Specifically, the $\mathrm{PHO}$ employed a clinical pharmacist to help create claims-based prescribing reports for PCPs generated from a relational (MS Access) database. Prescriberspecific reports were distributed to 57 PCPs operating under a global capitation contract with 9,046 enrolled members. A total of 795 high-cost prescriptions were identified, and 477 (60\%) letters were generated to patients for new (substituted) prescriptions. The most common drug class that underwent substitution was oral contraceptives (278 patients), followed by antihistamines (150) and antihyperlipidemics (20). The onetime start-up developments costs were reported as $\$ 35,000$, with annualized savings of $\$ 103,635$ - a 3-to-1 return on investment in the first year.

The report of the intervention in that academic PHO did not identify the time period and did not provide enough information to determine relative (percentage) savings. However, the reported savings per member can be derived as $\$ 11.46$ per-member-per-year (PMPY) or $\$ 0.95$ per-member-per-month. This study did employ comparison data from the remainder of the integrated delivery network that did not use the information intervention with PCPs and found the 22\% reduction in use of high-cost drugs to be greater than the comparison group $(P<0.001)$. Yet, it is noteworthy that the information intervention with PCPs coincided with a change in drug benefit design for the health plan members in which a copayment for third-tier (presumably nonformulary) drugs was added at $\$ 25$ per prescription. This 3-tier copayment design $(\$ 5$ for generic drugs, $\$ 10$ for tier-2 brand drugs, and $\$ 25$ for tier-3 brand drugs) replaced a 2 -tier ( $\$ 5$ generic and $\$ 10$ brand drug) copayment design. Implementation of a 3-tier drug benefit copay design has been shown to slow the rate of increase in total drug utilization by at least $40 \%$ versus a comparison group and reduce the utilization of tier-3 copay drugs by $18 \%$ compared with baseline (preintervention for the same group) and by 23\% versus the comparison group. ${ }^{3}$

In an article in this issue of the Journal, a clinical pharmacist intervention in a nonacademic primary care medical group operating under a global capitation agreement was successful in reducing the adverse drug cost trend that threatened the financial integrity of the medical group. ${ }^{4}$ The study by Walker and Willey did not have a control group but did use national data by drug class. The clinical pharmacist was hired by the medical group for a specific purpose: to reduce the increase in drug costs incurred by members enrolled in the health plan with the global capitation agreement. Cost outcomes were the principal focus of this intervention, but the authors found that the participating physicians reported no incidences of patient complaints or dissatisfaction associated with the medication therapy management intervention.

The study by Walker and Willey perhaps confirms what one would expect-that a clinical pharmacist employed by a medical group and armed with information about relative drug costs can guide physicians to selection of lower-cost therapeutic alternatives. The combination of a physician financial incentive (global capitation) and information (physician-specific prescribing information derived from pharmacy claims data) was aided further by employment of the clinical pharmacist by the medical group. Others have observed that staff pharmacists employed in staff-model health plans are more likely to be viewed as clinical partners and educators compared with independent physician associations (IPAs) in which pharmacists employed by the health plan or pharmacy benefit managers are more likely to be viewed as "regulators and cost cutters."

Walker and Willey differentiate the favorable effect on drug cost trend (from an increase in per-member costs of 31\% in the prior year to a decrease of $2 \%$ in the intervention year) between utilization and price and do so by drug class. The results are noteworthy. Antibiotic costs were reduced by $22 \%$ through a combination of the use of lower-cost therapeutic alternatives (15\% reduction in the average cost per antibiotic prescription claim) and $8 \%$ reduction in antibiotic utilization.

For the clinical conditions associated with cardiovascular disease, the clinical pharmacist intervention derived cost savings from drug cost rather than from reduction in utilization. A $24 \%$ increase in utilization of angiotensin-converting enzyme inhibitors (ACEI) was offset by a $26 \%$ reduction in the average cost per ACEI claim, resulting in lower ACEI costs PMPY. The use of calcium channel blockers (CCB) increased by 20\%, but PMPY costs for CCBs were reduced by a reduction in the average cost per CCB claim. The utilization of statins increased $16 \%$ in the medical group, but a reduction in the average cost per statin claim held the increase in statin costs to 10\% PMPY compared with the prior year.

At a time when proton pump inhibitors (PPI) were driving up the pharmacy costs for heartburn and gastro-esophageal reflux disease nationwide, this PCP medical group with clinical pharmacist intervention experienced a $9 \%$ increase in the combined utilization of PPIs and histamine 2 -blockers, similar to the national data, but the increased use of generic ranitidine helped to reduce the average cost per pharmacy claim, resulting in no increase in PMPY costs in the intervention year. One other study has been published from clinical pharmacist intervention in an academic IPA, ${ }^{6}$ but that report was primarily descriptive and did not identify the specific PMPY cost savings nor the effects of the clinical pharmacist intervention on either drug cost per unit (i.e., per prescription or per day of therapy) or utilization, by therapeutic class. 